\title{
Crossing Numbers and Parameterized Complexity
}

\author{
Michael J. Pelsmajer ${ }^{1}$, Marcus Schaefer ${ }^{2}$, and Daniel Stefankovič ${ }^{3}$ \\ ${ }^{1}$ Illinois Institute of Technology, Chicago, IL 60616, USA \\ pelsmajer@iit.edu \\ 2 DePaul University, Chicago, IL 60604, USA \\ mschaefer@cs.depaul.edu \\ ${ }^{3}$ University of Rochester, Rochester, NY 14627, USA \\ stefanko@cs.rochester.edu
}

\begin{abstract}
The odd crossing number of $G$ is the smallest number of pairs of edges that cross an odd number of times in any drawing of $G$. We show that there always is a drawing realizing the odd crossing number of $G$ that uses at most $9^{k}$ crossings, where $k$ is the odd crossing number of $G$. As a consequence of this and a result of Grohe we can show that the odd crossing number is fixed-parameter tractable.
\end{abstract}

\section{Introduction}

The crossing number of a graph $G$, denoted $\operatorname{cr}(G)$, is the smallest number of intersections in any drawing of $G$. There are many variants of this fundamental notion; in this paper we concentrate on the odd crossing number which counts pairs of edges that intersect an odd number of times. More formally, $\operatorname{ocr}(G)$ is the smallest number of pairs of edges in any drawing of $G$ that cross an odd number of times. Similarly, we can define the pair crossing number of $G, \operatorname{pcr}(G)$, as the smallest number of pairs of edges that intersect in any drawing of $G$. For historical background and summary on different notions of crossing numbers, see the paper by Pach and Tóth 4 .

From the definition we have

$$
\operatorname{ocr}(G) \leq \operatorname{pcr}(G) \leq \operatorname{cr}(G) .
$$

We also know that $\operatorname{cr}(G) \leq 2 \operatorname{ocr}(G)^{2}$ ([4], for a new proof, see [6]) and $\operatorname{cr}(G) \leq$ $2 \operatorname{pcr}(G)^{2} / \log ^{2} \operatorname{pcr}(G)[9]$. And while we do know that $\operatorname{ocr}(G) \neq \operatorname{cr}(G)$ in general [5], it is possible that $\operatorname{pcr}(G)=\operatorname{cr}(G)$ for all $G$.

This suggests the question of how close we can come to realizing this suspected equality in a drawing; that is, what can we say about the number of crossings needed in a pcr-optimal drawing? Maybe surprisingly, the best upper bounds we know are exponential 77 (see the end of Section 3 for a discussion).

To the extent that we believe that $\operatorname{pcr}(G)=\operatorname{cr}(G)$ this is a bit of an embarrassment, since the bound should be the identity. On the other hand, the pair crossing number does tie in very closely with the string graph problem, and a 
proof that $\operatorname{pcr}(G)=\operatorname{cr}(G)$ based on redrawing would have to change which pairs of edges intersect: if we restrict redrawing moves to those that do not change which pairs of edges intersect, there is an exponential separation between paircrossing number and crossing number due to due to Kratochvíl and Matoušek [3] (they phrased their example for string graphs).

In this paper we address the question of how many crossings are needed to realize an ocr-optimal drawing. We prove an exponential upper bound, similar to what was shown in the case of pcr. It is not inconceivable that the actual gap is exponential; this would be a very interesting result indeed.

Grohe showed that $\operatorname{cr}(G) \leq k$ can be decided in quadratic time for any fixed $k$ 1]. This means that the crossing number problem is fixed-parameter tractable: it can be solved in time $O\left(n^{c}\right)$ for some constant $c$ not depending on the parameter $k$. In Section 3 we show how to combine our exponential upper on crossings in an ocr-optimal drawing with Grohe's proof to conclude that ocr can also be decided in quadratic time. This result is somewhat unsatisfactory in that it relies on Grohe's proof rather than establishing a reduction that would allows us to transfer the fixed-parameter tractability result from cr to ocr automatically (such reductions are known as fpt-reductions). If we had such a reduction, Grohe's result could be replaced when a better fixed-parameter algorithm for crossing number is found. Indeed, Grohe's result has very recently been improved from quadratic to linear time by Kawarabayashi and Reed [2. Kawarabayashi and Reed also claim (albeit without supplying details) that ocr and pcr are fixed-parameter tractable. They do not have a reduction either, but have to verify that their constructions work for ocr and pcr in place of cr. We believe that their missing details can be filled in, for example, by using Theorem[1] and Theorem 3.2 from [7.

One motivation behind the introduction of the crossing number variants pcr and ocr was the hope that they would turn out to be easier objects to deal with than the crossing number itself. For example, the odd crossing number problem can be rephrased as a shortest vector problem in an appropriately chosen vector space. The hope remains that through these alternative approaches we might obtain feasible approximation algorithms or parameterized algorithms solving the crossing number problem (the results by Grohe, Kawarabayashi and Reed do not yield feasible algorithms).

\section{2 ocr-Critical Drawings}

In this section we show that a drawing of a graph realizing the odd crossing number has at most an exponential number of crossings.

Theorem 1. For any graph $G$ there is a drawing of $G$ with odd-crossing number $c=\operatorname{ocr}(G)$ and crossing number at most $9^{c}$.

The core of the proof is a redrawing idea: consider a drawing of $G$, and a particular edge $e$ of $G$. Imagine that $e$ is drawn as a horizontal line segment, and consider an arbitrary subsegment $I$. Consider the intersections of $e$ with other edges that occur within $I$. Without changing the odd-crossing number of the 
drawing, we can rearrange these intersections within $I$ such that for each edge $f \neq e$, the intersections of $f$ and $I$ are consecutive along $I$ : We can do this by simply pushing intersections to the left or the right. Whenever an intersection of $f$ with $e$ is pushed past an intersection of $f^{\prime}$ with $e$, it yields two new intersections between $f$ and $f^{\prime}$, which does not change the odd-crossing number of the drawing. Next, we claim that we can redraw $G$ such that each edge $f \neq e$ has at most 2 intersections with $I$, without changing the odd-crossing number of the drawing. Consider every edge $f$ that intersects $I$, one at a time. Split $f$ at each intersection with $I$, creating a set of curves $S_{I}$ with endpoints in $I$, except that two of the curves have one endpoint at an endpoint of $f$.

Let $\alpha$ and $\omega$ be the two curves in $S_{I}$ that have one end at an endpoint of $f$. Let $S_{\alpha}$ be the set of curves in $S_{I}$ that begin and end on the same side of $I$ where $\alpha$ ends. Let $S_{\alpha}^{\prime}$ be the set of curves in $S_{I}$ that begin and end on the other side of $I$, and let $S$ be the set of curves that begin and end at opposite sides of $I$.

Our goal is to reconnect the parts of $f$ so that the resulting curve traverses all of the original parts of $f$ except on a small neighborhood of $I$, and intersects $I$ at most twice. We proceed as follows: Start by following $\alpha$ from an endpoint of $f$ to its intersection with $I$. Continue by following all of the curves in $S_{\alpha}$, one after the other, then the curves of $S$, then the curves of $S_{\alpha}^{\prime}$ and end by following $\omega$ to the other endpoint of $f$. Move the endpoints of the curves at $I$ slightly, and connect consecutive curves in a small neighborhood of $I$ such that the resulting curve $f^{\prime}$ intersects $I$ as few times as possible. (For the moment, we ignore self-intersections of $f^{\prime}$.) The only steps at which intersecting $I$ may be unavoidable occur when going from $S$ to $S_{\alpha}^{\prime}$ and when going from $S_{\alpha}^{\prime}$ to $\omega$. Thus $f^{\prime}$ redraws $f$ using at most two intersections with $I$. Observe that the redrawing $f^{\prime}$ intersects $I$ exactly once if and only if either 1) $\alpha$ and $\omega$ approach $I$ from opposite sides and $|S|$ is even, or 2) $\alpha$ and $\omega$ approach $I$ from the same side and $|S|$ is odd. Before redrawing, the number of intersections between $f$ and $I$ is $1+2\left|S_{\alpha}\right|+|S|$ if $\alpha$ and $\omega$ approach $I$ from opposite sides and $2+2\left|S_{\alpha}\right|+|S|$ if $\alpha$ and $\omega$ approach $I$ from the same side. Thus, the number of intersections between $f$ and $I$ is odd if and only if the number of intersections between $f^{\prime}$ and $I$ is now one. Also, the parity of intersection of the redrawing $f^{\prime}$ with any other edge is the same as the parity of $f$ with that edge, since $f^{\prime}$ and $f$ agree except for in a small neighborhood of $I$, where $f$ intersects only $I$.

As we mentioned earlier, the redrawing $f^{\prime}$ might contain self-intersections, however, these can easily be removed (see [6], for example). Repeating this process for each edge that intersects $I$ results in at most $2 i$ intersections of edges with $I$, where $i$ is the number of edges $f \neq e$ that intersected $I$ an odd number of times before the redrawing.

We now apply this idea to bound the number of crossings necessary to realize a particular odd crossing number.

We begin with a drawing of $G$ achieving ocr $(G)$. Applying Theorem 2.1 from [6] allows us to assume that all even edges are without intersections. Then there are at most $k:=2 \operatorname{ocr}(G)$ edges, $e_{1}, \ldots, e_{k}$, involved in intersections in the drawing of $G$ under consideration. We will redraw these edges such that 
for $1 \leq i<j \leq k$, the number of intersections between $e_{i}$ and $e_{j}$ is at most $2\left(3^{i-1}\right)$. We redraw the edges in order, as follows: Begin by applying the procedure described earlier to $e_{1}$; then each other edge intersects $e_{1}$ at most twice, as desired. We want to keep the intersections along $e_{1}$ now, so we should not apply our procedure to subsequent edges. Instead, during the $j$ th step we split $e_{j}$ into segments at every intersection with an edge $e_{i}$ with $i<j$, and apply the procedure to each of those segments.

By induction, the number of intersections of $e_{j}$ and all $e_{i}$ with $i<j$ is at most $\sum_{i=1}^{j-1} 2\left(3^{i-1}\right)$, which equals $3^{j-1}-1$. Hence $e_{j}$ is split up into at most $3^{j-1}$ segments, and after applying the procedure to each segment, each $e_{i}$ with $i>j$ has at most $2\left(3^{j-1}\right)$ intersections with $e_{j}$, as desired.

The total number of crossings is $\sum_{1 \leq i<j \leq k} 2\left(3^{i-1}\right)$, or $\sum_{j=1}^{k} \sum_{i=1}^{j-1} 2\left(3^{i-1}\right)=$ $\sum_{j=1}^{k}\left(3^{j-1}-1\right) \leq 3^{k}$.

\section{The Parameterized Complexity of ocr}

In this section we will derive a quadratic time algorithm for computing ocr by adapting Grohe's result [1.

Grohe showed that for a fixed $k$ it can be decided in quadratic time whether the crossing number of a graph $G$ is at most $k$ [1. Grohe's algorithm proceeds as follows: for some function $w(k)$ only depending on $k$ it tests whether the tree-width of $G$ is at most $w(k)$; if that is not the case, then either the crossing number of $G$ is larger than $k$ or we can find a part of $G$ that is not involved in any crossing in a cr-optimal drawing. If the crossing number is larger than $k$, we are done; otherwise we can replace $G$ with a smaller graph and keep track of its crossing-free part. Repeating this procedure we will eventually reach a graph of bounded tree-width for which we can decide whether $\operatorname{cr}(G) \leq k$ using Courcelle's theorem (details to be explained below).

This central result of Grohe's paper is contained in his Corollary 8 [1] which we reproduce nearly verbatim below. Here, a $k$-good drawing with respect to $F$ of $G$ is a drawing of $G$ with crossing number at most $k$ in which none of the edges of $F$ are involved in a crossing.

Proposition 1 (Grohe [1]). There is a quadratic time algorithm that, given a graph $G$ and an edge set $F \subseteq E(G)$, either recognizes that the crossing number of $G$ is greater than $k$ or computes a graph $G^{\prime}$ and an edge set $F^{\prime} \subseteq E\left(G^{\prime}\right)$ such that the tree-width of $G^{\prime}$ is at most $w(k)$ and $G$ has a $k$-good drawing with respect to $F$ if and only if $G^{\prime}$ has a k-good drawing with respect to $F^{\prime}$.

We cannot immediately apply Grohe's result as stated to help us settle the parameterized complexity of computing the odd crossing number, since it is not clear how the odd crossing number of $G^{\prime}$ (with the planarity restriction on $F^{\prime}$ ) relates to the odd crossing number of $G$ (with the planarity restriction on $F$ ). Fortunately, a closer look at Grohe's proof shows that a stronger version of the proposition is true.

For a graph $G$ let a $(k, \ell)$-good drawing with respect to $F$ be a drawing of $G$ with crossing number at most $k$ and odd crossing number at most $\ell$ in which 
none of the edges of $F$ are involved in any crossings. An inspection of Grohe's proof of his Corollary 8 shows that it is true for $(k, \ell)$-good drawing in place of $k$-good drawings. The reason is that in the core step of the proof [1, Lemma 5] the redrawing is local and does not increase the odd crossing number.

Lemma 1. There is a quadratic time algorithm that, given a graph $G$ and an edge set $F \subseteq E(G)$, either recognizes that the crossing number of $G$ is greater than $k$ or computes a graph $G^{\prime}$ and an edge set $F^{\prime} \subseteq E\left(G^{\prime}\right)$ such that the treewidth of $G^{\prime}$ is at most $w(k)$ and $G$ has a $(k, \ell)$-good drawing with respect to $F$ if and only if $G^{\prime}$ has a $(k, \ell)$-good drawing with respect to $F^{\prime}$.

By Theorem 1 $G$ has odd crossing number at most $k$ if and only if $G$ has a $\left(9^{k}, k\right)$-good drawing with respect to $F=\emptyset$. We can now proceed as in Grohe's algorithm to look for such a drawing of $G$. We either find that the crossing number of $G$ is larger than $9^{k}$, which implies that the odd crossing number is larger than $k$ (actually, much larger by the quadratic bound between odd crossing number and crossing number due to Pach and Tóth 4]) or we obtain a graph $G^{\prime}$ of tree-width at most $w(k)$ and an edge set $F^{\prime}$ such that $G$ has odd crossing number at most $k$ if and only if $G^{\prime}$ has a $\left(9^{k}, k\right)$-good drawing in which none of the edges of $F^{\prime}$ are involved in an intersection.

If we can now show that "having a $\left(9^{k}, k\right)$-good drawing with respect to $F$ " can be expressed in the second-order monadic logic of graphs, we can apply Courcelle's theorem which states that formulas of second-order monadic logic can be decided in linear time for graphs of bounded tree-width (remember that the tree-width $w(k)$ of $G^{\prime}$ depends on $k$ only, and is therefore considered fixed). Consider a $\left(9^{k}, k\right)$-good drawing of $G$ if it exists. Replacing every crossing with a new vertex yields a planar drawing; adding four more vertices and edges around this vertex we can ensure that a planar drawing of the resulting graph corresponds to a $\left(9^{k}, k\right)$-good drawing of $G$. (See Figure 1)
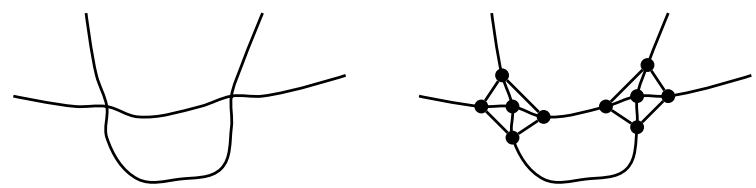

Fig. 1. Two crossings, before (left) and after (right)

Using monadic second order logic we can specify a set of at most $2 k$ edges (not in $F$ ) and subdivide each of those $2 k$ edges $3\left(9^{k}\right)$ times. These subdivided edges can now be used to express that there is a $\left(9^{k}, k\right)$-good drawing of $G$ with respect to $F$ : We can express that the $i$ th intersection along edge $e$ is also the $j$ th intersection along edge $f$ by identifying the $3 i-1$ st vertex along the subdivided $e$ with the $3 j-1$ st vertex along the subdivided $f$ and adding edges between vertices $3 i-2$ and $3 i$ on $e$ and $f$ to build the 4-cycle in the right half of Figure 1 to ensure $e$ and $f$ actually cross (rather than just touch) at their intersection point. Using this, we can write down explicitly a formula describing the order in which 
edges cross every particular edge. While this leads to a formula exponentially large in $9^{k}$, this is not a problem, since $k$ is fixed. Since we are specifying how the crossings occur explicitly, we can restrict ourselves to those formulas describing a drawing with odd crossing number at most $k$.

Theorem 2. For a fixed $k$ we can decide $\operatorname{ocr}(G) \leq k$ in quadratic time.

What about the pair-crossing number? A drawing of a graph can always be redrawn without making two pairs of edges intersect that did not intersect in the original drawing while reducing the crossing number of the drawing to at most $k 2^{k}$ (where $k$ is the number of edges involved in crossings) [7. If we start with a drawing that realizes the pair-crossing number of the graph, this shows that we can always assume that a pair-crossing critical drawing has crossing number at most $k 2^{k}$. With this result we can repeat the argument we used for odd crossing numbers, allowing us to conclude that the pair crossing number is fixed-parameter tractable.

Theorem 3. For a fixed $k$ we can decide $\operatorname{pcr}(G) \leq k$ in quadratic time.

Acknowledgments. We would like to thank Martin Grohe for suggesting this problem, and Petr Hliněný for helpful discussions.

\section{References}

1. Grohe, M.: Computing crossing numbers in quadratic time. In: Proceedings of the 32nd ACM Symposium on Theory of Computing, pp. 231-236 (July 6-8, 2001)

2. Kawarabayashi, K.i., Reed, B.: Computing crossing number in linear time. STOC (accepted, 2007)

3. Kratochvíl, J., Matoušek, J.: String graphs requiring exponential representations. Journal of Combinatorial Theory, Series B 53, 1-4 (1991)

4. Pach, J., Tóth, G.: Which crossing number is it anyway? J. Combin. Theory Ser. B 80(2), 225-246 (2000)

5. Pelsmajer, M.J., Schaefer, M., Štefankovič, D.: Odd crossing number is not crossing number. In: Healy, P., Nikolov, N.S. (eds.) GD 2005. LNCS, vol. 3843, pp. 386-396. Springer, Heidelberg (2006)

6. Pelsmajer, M.J., Schaefer, M., Štefankovič, D.: Removing even crossings. In: Felsner, S. (ed.) EuroComb 2005. 2005 European Conference on Combinatorics, Graph Theory and Applications of DMTCS Proceedings. Discrete Mathematics and Theoretical Computer Science, vol. AE, pp. 105-109 (April 2005)

7. Schaefer, M., Štefankovič, D.: Decidability of string graphs. In: STOC-2001. Proceedings of the 33th Annual ACM Symposium on Theory of Computing, pp. 241-246 (2001)

8. Tóth, G.: Note on the pair-crossing number and the odd-crossing number (Unpublished manuscript)

9. Valtr, P.: On the pair-crossing number. In: Combinatorial and Computational Geometry. Math. Sci. Res. Inst. Publ. vol. 52, pp. 569-575. Cambridge Univ. Press, Cambridge (2005) 